\title{
Existence of Positive Solutions for Fractional Differential Equation with Nonlocal Boundary Condition
}

\author{
Hongliang Gao and Xiaoling Han \\ Department of Mathematics, Northwest Normal University, Lanzhou 730070, China \\ Correspondence should be addressed to Xiaoling Han, hanxiaoling@nwnu.edu.cn
}

Received 29 May 2011; Revised 24 August 2011; Accepted 28 September 2011

Academic Editor: Om Agrawal

Copyright (C) 2011 H. Gao and X. Han. This is an open access article distributed under the Creative Commons Attribution License, which permits unrestricted use, distribution, and reproduction in any medium, provided the original work is properly cited.

By using the fixed point theorem, existence of positive solutions for fractional differential equation with nonlocal boundary condition $D_{0+}^{\alpha} u(t)+a(t) f(t, u(t))=0,0<t<1, u(0)=0, u(1)=$ $\sum_{i=1}^{\infty} \alpha_{i} u\left(\xi_{i}\right)$ is considered, where $1<\alpha \leq 2$ is a real number, $D_{0+}^{\alpha}$ is the standard Riemann-Liouville differentiation, and $\xi_{i} \in(0,1), \alpha_{i} \in[0, \infty)$ with $\sum_{i=1}^{\infty} \alpha_{i} \xi_{i}^{\alpha-1}<1, a(t) \in C([0,1],[0, \infty)), f(t, u) \in$ $C([0,1] \times[0, \infty),[0, \infty))$.

\section{Introduction}

Fractional differential equations have been of great interest recently. It is caused both by the intensive development of the theory of fractional calculus itself and by the applications of such constructions in various sciences such as physics, mechanics, chemistry, and engineering. For details, see [1-6] and references therein.

It should be noted that most of papers and books on fractional calculus are devoted to the solvability of linear initial fractional differential equations in terms of special functions [68]. Recently, there are some papers that deal with the existence and multiplicity of solution (or positive solution) of nonlinear initial fractional differential equation by the use of techniques of nonlinear analysis (fixed-point theorems, Leray-Shauder theory, etc.); see [9-17].

Recently, Bai and Lü [15] studied the existence of positive solutions of nonlinear fractional differential equation

$$
\begin{gathered}
D_{0+}^{\alpha} u(t)+f(t, u(t))=0, \quad 0<t<1, \\
u(0)=u(1)=0,
\end{gathered}
$$


where $1<\alpha \leq 2$ is a real number, $D_{0+}^{\alpha}$ is the standard Riemann-Liouville differentiation, and $f:[0,1] \times[0, \infty) \rightarrow[0, \infty)$ is continuous.

In this paper, we study the existence of positive solutions for fractional differential equation with nonlocal boundary condition

$$
\begin{aligned}
D_{0+}^{\alpha} u(t)+a(t) f(t, u(t)) & =0, \quad 0<t<1, \\
u(0)=0, \quad u(1) & =\sum_{i=1}^{\infty} \alpha_{i} u\left(\xi_{i}\right),
\end{aligned}
$$

where $1<\alpha \leq 2$ is a real number, $D_{0+}^{\alpha}$ is the standard Riemann-Liouville differentiation, and $\xi_{i} \in(0,1), \alpha_{i} \in[0, \infty)$ with $\sum_{i=1}^{\infty} \alpha_{i} \xi_{i}^{\alpha-1}<1, a(t) \in C([0,1],[0, \infty)), f(t, u) \in C([0,1] \times$ $[0, \infty),[0, \infty))$.

We assume the following conditions hold throughout the paper:

(H1) $\xi_{i} \in(0,1), \alpha_{i} \in[0, \infty)$ is both constants with $\sum_{i=1}^{\infty} \alpha_{i} \xi_{i}^{\alpha-1}<1$,

$(\mathrm{H} 2) a(t) \in C([0,1],[0, \infty)), a(t) \not \equiv 0$ on $[a, b] \subset(0,1)$,

(H3) $f(t, u) \in C([0,1] \times[0, \infty),[0, \infty))$.

Remark 1.1. To our knowledge, there are no results about the existence of positive solutions for problem (1.2).

\section{The Preliminary Lemmas}

For the convenience of the reader, we present here the necessary definitions from fractional calculus theory. These definitions can be found in the recent literature.

Definition 2.1. The fractional integral of order $\alpha>0$ of a function $y:(0, \infty) \rightarrow R$ is given by

$$
I_{0+}^{\alpha} y(t)=\frac{1}{\Gamma(\alpha)} \int_{0}^{t}(t-s)^{\alpha-1} y(s) d s
$$

provided the right side is pointwise defined on $(0, \infty)$.

Definition 2.2. The fractional derivative of order $\alpha>0$ of a function $y:(0, \infty) \rightarrow R$ is given by

$$
D_{0+}^{\alpha} y(t)=\frac{1}{\Gamma(n-\alpha)}\left(\frac{d}{d t}\right)^{n} \int_{0}^{t} \frac{y(s)}{(t-s)^{\alpha-n+1}} d s,
$$

where $n=[\alpha]+1$, provided the right side is pointwise defined on $(0, \infty)$.

Definition 2.3. The map $\theta$ is said to be a nonnegative continuous concave functional on a cone $P$ of a real Banach space $E$, provided that $\theta: P \rightarrow[0, \infty)$ is continuous and

$$
\theta(t x+(1-t) y) \geq t \theta(x)+(1-t) \theta(y),
$$

for all $x, y \in P$ and $0 \leq t \leq 1$. 
Remark 2.4. As a basic example, we quote for $\lambda>-1$,

$$
D_{0+}^{\alpha} t^{\lambda}=\frac{\Gamma(\lambda+1)}{\Gamma(\lambda-\alpha+1)} t^{\lambda-\alpha},
$$

giving in particular $D_{0+}^{\alpha} t^{\alpha-m}=0, m=1,2, \ldots, N$, where $N$ is the smallest integer greater than or equal to $\alpha$.

From Definition 2.2 and Remark 2.4, we then obtain the following.

Lemma 2.5. Let $\alpha>0$. If one assumes $u \in C(0,1) \cap L(0,1)$, then the fractional differential equation

$$
D_{0+}^{\alpha} u(t)=0
$$

has $u(t)=C_{1} t^{\alpha-1}+C_{2} t^{\alpha-2}+\cdots+C_{N} t^{\alpha-N}, C_{i} \in R, i=1,2, \ldots, N$, where $N$ is the smallest integer greater than or equal to $\alpha$, as unique solutions.

Lemma 2.6. Assume that $u \in C(0,1) \cap L(0,1)$ with a fractional derivative of order $\alpha>0$ that belongs to $u \in C(0,1) \cap L(0,1)$. Then,

$$
I_{0+}^{\alpha} D_{0+}^{\alpha} u(t)=u(t)+C_{1} t^{\alpha-1}+C_{2} t^{\alpha-2}+\cdots+C_{N} t^{\alpha-N},
$$

for some $C_{i} \in R, i=1,2, \ldots, N$.

Lemma 2.7 (see [15]). Given $y \in C[0,1]$ and $1<\alpha \leq 2$, the unique solution of

$$
\begin{gathered}
D_{0+}^{\alpha} u(t)+y(t)=0, \quad 0<t<1, \\
u(0)=u(1)=0
\end{gathered}
$$

is

$$
u(t)=\int_{0}^{1} G(t, s) y(s) d s,
$$

where

$$
G(t, s)= \begin{cases}\frac{[t(1-s)]^{\alpha-1}-(t-s)^{\alpha-1}}{\Gamma(\alpha)}, & 0 \leq s \leq t \leq 1 \\ \frac{[t(1-s)]^{\alpha-1}}{\Gamma(\alpha)}, & 0 \leq t \leq s \leq 1\end{cases}
$$

Lemma 2.8. Suppose $(H 1)$ holds. Given $y \in C[0,1]$ and $1<\alpha \leq 2$, the unique solution of

$$
\begin{aligned}
& D_{0+}^{\alpha} u(t)+y(t)=0, \quad 0<t<1, \\
& u(0)=0, \quad u(1)=\sum_{i=1}^{\infty} \alpha_{i} u\left(\xi_{i}\right)
\end{aligned}
$$


is

$$
u(t)=\int_{0}^{1} G(t, s) y(s) d s+B(y) t^{\alpha-1}
$$

where

$$
\begin{gathered}
G(t, s)= \begin{cases}\frac{[t(1-s)]^{\alpha-1}-(t-s)^{\alpha-1}}{\Gamma(\alpha)}, & 0 \leq s \leq t \leq 1, \\
\frac{[t(1-s)]^{\alpha-1}}{\Gamma(\alpha)}, & 0 \leq t \leq s \leq 1,\end{cases} \\
B(y)=\frac{\sum_{i=1}^{\infty} \alpha_{i} \int_{0}^{1} G\left(\xi_{i}, s\right) y(s) d s}{1-\sum_{i=1}^{\infty} \alpha_{i} \xi_{i}^{\alpha-1}} .
\end{gathered}
$$

Proof. By applying Lemmas 2.6 and 2.7, we have

$$
u(t)=\int_{0}^{1} G(t, s) y(s) d s+C_{1} t^{\alpha-1}+C_{2} t^{\alpha-2}
$$

Because

$$
\sum_{i=1}^{\infty} \alpha_{i} \int_{0}^{1} G\left(\xi_{i}, s\right) d s=\frac{\sum_{i=1}^{\infty} \alpha_{i} \xi_{i}^{\alpha-1}\left(1-\xi_{i}\right)}{\alpha \Gamma(\alpha)}, \quad \alpha_{i} \xi_{i}^{\alpha-1}\left(1-\xi_{i}\right)<\alpha_{i} \xi_{i}^{\alpha-1}
$$

by $(\mathrm{H} 1), \sum_{i=1}^{\infty} \alpha_{i} \xi_{i}^{\alpha-1}\left(1-\xi_{i}\right)$ is converge. Therefore, $\sum_{i=1}^{\infty} \alpha_{i} \int_{0}^{1} G\left(\xi_{i}, s\right) d s$ is converge. $y(t)$ is continuous function on $[0,1]$, so $\sum_{i=1}^{\infty} \alpha_{i} \int_{0}^{1} G\left(\xi_{i}, s\right) y(s) d s$ is converge.

By $u(0)=0, u(1)=\sum_{i=1}^{\infty} \alpha_{i} u\left(\xi_{i}\right)$, there are $C_{2}=0, C_{1}=\left(\sum_{i=1}^{\infty} \alpha_{i} \int_{0}^{1} G\left(\xi_{i}, s\right) y(s) d s\right) /(1-$ $\left.\sum_{i=1}^{\infty} \alpha_{i} \xi_{i}^{\alpha-1}\right)$. Therefore,

$$
\begin{aligned}
u(t) & =\int_{0}^{1} G(t, s) y(s) d s+B(y) t^{\alpha-1}, \\
B(y) & =\frac{\sum_{i=1}^{\infty} \alpha_{i} \int_{0}^{1} G\left(\xi_{i}, s\right) y(s) d s}{1-\sum_{i=1}^{\infty} \alpha_{i} \xi_{i}^{\alpha-1}} .
\end{aligned}
$$

Lemma 2.9 (see [15]). The function $G(t, s)$ defined by (2.9) satisfies the following conditions:

(1) $G(t, s)>0$, for $t, s \in(0,1)$,

(2) there exists a positive function $\gamma \in C(0,1)$ such that

$$
\min _{(1 / 4) \leq t \leq(3 / 4)} G(t, s) \geq \gamma(s) \max _{0 \leq t \leq 1} G(t, s)=\gamma(s) G(s, s), \quad 0<s<1
$$


Lemma 2.10 (see [18]). Let $E$ be a Banach space, $P \subseteq E$ a cone and $\Omega_{1}, \Omega_{2}$ two bounded open sets of E with $0 \in \Omega_{1} \subset \bar{\Omega}_{1} \subset \Omega_{2}$. Suppose that $A: P \cap\left(\bar{\Omega}_{2} \backslash \Omega_{1}\right) \rightarrow P$ is a completely continuous operator such that either

(i) $\|A x\| \leq\|x\|, x \in P \cap \partial \Omega_{1}$ and $\|A x\| \geq\|x\|, x \in P \cap \partial \Omega_{2}$, or

(ii) $\|A x\| \geq\|x\|, x \in P \cap \partial \Omega_{1}$ and $\|A x\| \leq\|x\|, x \in P \cap \partial \Omega_{2}$

holds. Then, A has a fixed point in $P \cap\left(\bar{\Omega}_{2} \backslash \Omega_{1}\right)$.

Lemma 2.11 (see [19]). Let $P$ be a cone in real Banach space $E, P_{c}=\{x \in P \mid\|x\| \leq c\}, \theta$ a nonnegative continuous concave functional on $P$ such that $\theta(x) \leq\|x\|$, for all $x \in \bar{P}_{c}$, and $P(\theta, b, d)=\{x \in P \mid b \leq \theta(x),\|x\| \leq d\}$. Suppose that $A: \bar{P}_{c} \rightarrow \bar{P}_{c}$ is completely continuous, and there exist constants $0<a<b<d \leq c$ such that

(C1) $\{x \in P(\theta, b, d) \mid \theta(x)>b\} \neq \emptyset$, and $\theta(A x)>b, x \in P(\theta, b, d)$,

(C2) $\|A x\| \leq a$, for $x \leq a$,

(C3) $\theta(A x)>$ for $x \in P(\theta, b, c)$ with $\|A x\|>d$.

Then, $A$ has at least three fixed points $x_{1}, x_{2}, x_{3}$ with

$$
\left\|x_{1}\right\|<a, \quad b<\theta\left(x_{2}\right), \quad a<\left\|x_{3}\right\|, \quad \theta\left(x_{3}\right)<b .
$$

Remark 2.12. If there holds $d=c$, then condition (C1) of Lemma 2.11 implies condition (C3) of Lemma 2.11.

\section{The Main Results}

Let $E=C[0,1]$ be endowed with the ordering $u \leq v$ if $u(t) \leq v(t)$ for all $t \in[0,1]$, and the maximum norm, $\|u\|=\max _{0 \leq t \leq 1}|u(t)|$. Define the cone $P \subset E$ by $P=\{u \in E \mid u(t) \geq 0\}$.

Let the nonnegative continuous concave functional $\theta$ on the cone $P$ be defined by $\theta(u)=\min _{(1 / 4) \leq t \leq(3 / 4)}|u(t)|$.

Lemma 3.1 (see [15]). Let $T: P \rightarrow E$ be the operator defined by $T u(t):=\int_{0}^{1} G(t, s) f(s, u(s)) d s$, then $T: P \rightarrow P$ is completely continuous.

Lemma 3.2. Let $A: P \rightarrow E$ be the operator defined by

$$
A u(t):=\int_{0}^{1} G(t, s) a(s) f(s, u(s)) d s+B(a(\cdot) f(\cdot, u(\cdot))) t^{\alpha-1},
$$

then $A: P \rightarrow P$ is completely continuous. 
Proof. The proof is similar to Lemma 3.1, so we omit.

Denote

$$
\begin{gathered}
M=\left(\int_{0}^{1} G(s, s) a(s) d s+\frac{\sum_{i=1}^{\infty} \alpha_{i} \int_{0}^{1} G\left(\xi_{i}, s\right) a(s) d s}{1-\sum_{i=1}^{\infty} \alpha_{i} \xi_{i}^{\alpha-1}}\right)^{-1}, \\
N=\left(\int_{1 / 4}^{3 / 4} \gamma(s) G(s, s) a(s) d s\right)^{-1} .
\end{gathered}
$$

Theorem 3.3. Assume (H1)-(H3) hold, and there exist two positive constants $r_{2}>r_{1}>0$ such that

(1) $f(t, u) \leq M r_{2}$, for all $t \in[0,1], u \in\left[0, r_{2}\right]$,

(2) $f(t, u) \geq N r_{1}$, for all $t \in[0,1], u \in\left[0, r_{1}\right]$, where $M, N$ is defined in $(*)$,

then problem (1.2) has at least one positive solution $u$ such that $r_{1} \leq\|u\| \leq r_{2}$.

Proof. By Lemmas 2.8 and 3.2, we know $A: P \rightarrow P$ is completely continuous, and problem (1.2) has a solution $u=u(t)$ if and only if $u$ solves the operator equation $u=A u$. In order to apply Lemma 2.10, we separate the proof into the following two steps.

Step 1. Let $\Omega_{2}=\left\{u \in P \mid\|u\| \leq r_{2}\right\}$. For $u \in \partial \Omega_{2}$, we have $0 \leq u(t) \leq r_{2}$ for all $t \in[0,1]$. It follows from (1) that for $t \in[0,1]$,

$$
\begin{aligned}
\|A u\| & \leq \int_{0}^{1} G(s, s) a(s) f(s, u(s)) d s+\frac{\sum_{i=1}^{\infty} \alpha_{i} \int_{0}^{1} G\left(\xi_{i}, s\right) a(s) f(s, u(s)) d s}{1-\sum_{i=1}^{\infty} \alpha_{i} \xi_{i}^{\alpha-1}} \\
& \leq M r_{2}\left[\int_{0}^{1} G(s, s) a(s) d s+\frac{\sum_{i=1}^{\infty} \alpha_{i} \int_{0}^{1} G\left(\xi_{i}, s\right) a(s) d s}{1-\sum_{i=1}^{\infty} \alpha_{i} \xi_{i}^{\alpha-1}}\right] \\
& =r_{2}=\|u\| .
\end{aligned}
$$

Therefore,

$$
\|A u\| \leq\|u\|, \quad u \in P \cap \partial \Omega_{2}
$$

Step 2. Let $\Omega_{1}=\left\{u \in P \mid\|u\| \leq r_{1}\right\}$. For $u \in \partial \Omega_{1}$, we have $0 \leq u(t) \leq r_{1}$ for all $t \in[0,1]$. By assumption (2), for $t \in[1 / 4,3 / 4]$, there is

$$
\begin{aligned}
(A u)(t) & =\int_{0}^{1} G(t, s) a(s) f(s, u(s)) d s+\frac{t^{\alpha-1} \sum_{i=1}^{\infty} \alpha_{i} \int_{0}^{1} G\left(\xi_{i}, s\right) a(s) f(s, u(s)) d s}{1-\sum_{i=1}^{\infty} \alpha_{i} \xi_{i}^{\alpha-1}} \\
& \geq \int_{0}^{1} r(s) G(s, s) a(s) f(s, u(s)) d s \\
& \geq N r \int_{1 / 4}^{3 / 4} r(s) G(s, s) a(s) d s \\
& =r_{1}=\|u\| .
\end{aligned}
$$


So,

$$
\|A u\| \geq\|u\|, \quad u \in P \cap \partial \Omega_{1} .
$$

Therefore, by (ii) of Lemma 2.10, we complete the proof.

Example 3.4. Consider the problem

$$
\begin{gathered}
D_{0+}^{3 / 2} u(t)+u^{2}+\frac{\sin t}{4}+\frac{1}{5}=0, \quad 0<t<1, \\
u(0)=0, \quad u(1)=\sum_{i=1}^{\infty} \alpha_{i} u\left(\xi_{i}\right),
\end{gathered}
$$

where $\sum_{i=1}^{\infty} \alpha_{i} \xi_{i}^{1 / 2}=1 / 5$.

A simple computation showed $M \geq 1.4, N \approx 13.6649$. Choosing $r_{1}=(1 / 70), r_{2}=$ $(9 / 10)$, we have

$$
\begin{gathered}
f(t, u)=u^{2}+\frac{\sin t}{4}+\frac{1}{5} \leq 1.2207 \leq M r_{2}, \quad(t, u) \in[0,1] \times\left[0, \frac{9}{10}\right] \\
f(t, u)=u^{2}+\frac{\sin t}{4}+\frac{1}{5} \geq \frac{1}{5} \geq N r_{1}, \quad(t, u) \in[0,1] \times\left[0, \frac{1}{70}\right] .
\end{gathered}
$$

With the use of Theorem 3.3, problem (3.6) has at least one positive solutions $u$ such that $(1 / 70) \leq\|u\| \leq(9 / 10)$.

Theorem 3.5. Assume (H1)-(H3) hold, and there exist constants $0<a<b<c$ such that the following assumptions hold:

(A1) $f(t, u)<M a$ for $(t, u) \in[0,1] \times[0, a]$,

(A2) $f(t, u) \geq N b$ for $(t, u) \in[1 / 4,3 / 4] \times[b, c]$,

(A3) $f(t, u) \leq M c$ for $(t, u) \in[0,1] \times[0, c]$, where $M, N$ is defined in $(*)$.

Then, the boundary value problem (1.2) has at least three positive solutions $u_{1}, u_{2}, u_{3}$ with

$$
\begin{gathered}
\left\|u_{1}\right\|<a, \quad b<\min _{(1 / 4) \leq t \leq(3 / 4)}\left|u_{2}\right|<\left\|u_{2}\right\| \leq c, \\
a<\left\|u_{3}\right\| \leq c, \quad \min _{(1 / 4) \leq t \leq(3 / 4)}\left|u_{3}\right|<b .
\end{gathered}
$$


Proof. We show that all the conditions of Lemma 2.9 are satisfied.

If $u \in \bar{P}_{c}$, then $\|u\| \leq c$. Assumption (A3) implies $f(t, u(t)) \leq M_{c}$ for $0 \leq t \leq 1$. Consequently,

$$
\begin{aligned}
\|A u\| & =\max _{0 \leq t \leq 1}\left|\int_{0}^{1} G(t, s) a(s) f(s, u(s)) d s+\frac{t^{\alpha-1} \sum_{i=1}^{\infty} \alpha_{i} \int_{0}^{1} G\left(\xi_{i}, s\right) a(s) f(s, u(s)) d s}{1-\sum_{i=1}^{\infty} \alpha_{i} \xi_{i}^{\alpha-1}}\right| \\
& \leq \int_{0}^{1} G(s, s) a(s) f(s, u(s)) d s+\frac{t^{\alpha-1} \sum_{i=1}^{\infty} \alpha_{i} \int_{0}^{1} G\left(\xi_{i}, s\right) a(s) f(s, u(s)) d s}{1-\sum_{i=1}^{\infty} \alpha_{i} \xi_{i}^{\alpha-1}} \\
& \leq\left[\int_{0}^{1} G(s, s) a(s) d s+\frac{\sum_{i=1}^{\infty} \alpha_{i} \int_{0}^{1} G\left(\xi_{i}, s\right) a(s) d s}{1-\sum_{i=1}^{\infty} \alpha_{i} \xi_{i}^{\alpha-1}}\right]\|u\| \\
& \leq\|u\| .
\end{aligned}
$$

Hence, $A: \bar{P}_{c} \rightarrow \bar{P}_{c}$. In the same way, if $u \in \bar{P}_{a}$, then assumption (A1) yields $f(t, u(t))<$ $M a, 0 \leq t \leq 1$. Therefore, condition (C2) of Lemma 2.11 is satisfied.

To check condition (C1) of Lemma 2.11, we choose $u(t)=(b+c) / 2,0 \leq t \leq 1$. It is easy to see that $u(t)=(b+c) / 2 \in P(\theta, b, c), \theta(u)=(\theta(b+c)) / 2>b$, and consequently, $\{u \in P(\theta, b, d) \mid \theta(u)>b\} \neq \emptyset$ Hence, if $u \in P(\theta, b, c)$, then $b \leq u(t) \leq c$ for $(1 / 4) \leq t \leq(3 / 4)$. From assumption (A2), we have $f(t, u(t)) \geq N b$ for $(1 / 4) \leq t \leq(3 / 4)$. So,

$$
\begin{aligned}
\theta(A u) & =\min _{(1 / 4) \leq t \leq(3 / 4)}|(A u)(t)| \\
& \geq \int_{0}^{1} \gamma(s) G(s, s) a(s) f(s, u(s)) d s \\
& >N b \int_{1 / 4}^{3 / 4} \gamma(s) G(s, s) a(s) d s \\
& =b=\|u\|
\end{aligned}
$$

$\theta(A u)>b$, for all $u \in P(\theta, b, c)$.

This shows that condition (C1) of Lemma 2.11 is also satisfied.

By Lemma 2.11 and Remark 2.12, the boundary value problem (1.2) has at least three positive solutions $u_{1}, u_{2}$, and $u_{3}$ with

$$
\begin{aligned}
& \left\|u_{1}\right\|<a, \quad b<\min _{(1 / 4) \leq t \leq(3 / 4)}\left|u_{2}\right|, \\
& a<\left\|u_{3}\right\|, \quad \min _{(1 / 4) \leq t \leq(3 / 4)}\left|u_{3}\right|<b .
\end{aligned}
$$

The proof is complete. 
Example 3.6. Consider the problem

$$
\begin{gathered}
D_{0+}^{3 / 2} u(t)+f(t, u)=0, \quad 0<t<1, \\
u(0)=0, \quad u(1)=\sum_{i=1}^{\infty} \alpha_{i} u\left(\xi_{i}\right)
\end{gathered}
$$

where $\sum_{i=1}^{\infty} \alpha_{i} \xi_{i}^{1 / 2}=(1 / 5)$,

$$
f(t, u)= \begin{cases}\left(\frac{t}{40}\right)+14 u^{2}, & u \leq 1 \\ 13+\left(\frac{t}{40}\right)+u, & u>1\end{cases}
$$

We have $M \geq 1.4, N \approx 13.6649$. Choosing $a=(1 / 14), b=1, c=36$, there hold

$$
\begin{gathered}
f(t, u)=\frac{t}{40}+14 u^{2} \leq 0.097 \leq M a, \quad(t, u) \in[0,1] \times\left[0, \frac{1}{14}\right], \\
f(t, u)=13+\frac{t}{40}+u \geq 14.025 \geq N b \approx 13.7, \quad(t, u) \in\left[\frac{1}{4}, \frac{3}{4}\right] \times[1,36], \\
f(t, u) \leq 13+\frac{t}{40}+u \leq 48.025 \leq M c \approx 50.4, \quad(t, u) \in[0,1] \times[0,36] .
\end{gathered}
$$

With the use of Theorem 3.5, problem (3.12) has at least three positive solutions $u_{1}, u_{2}$, and $u_{3}$ with

$$
\begin{aligned}
\max _{0 \leq t \leq 1}\left|u_{1}(t)\right| & <\frac{1}{14}, \quad 1<\min _{(1 / 4) \leq t \leq(3 / 4)}\left|u_{2}(t)\right|<\max _{0 \leq t \leq 1}\left|u_{2}(t)\right| \leq 36, \\
\frac{1}{14} & <\max _{0 \leq t \leq 1}\left|u_{3}(t)\right| \leq 36, \quad \min _{(1 / 4) \leq t \leq(3 / 4)}\left|u_{3}(t)\right|<1 .
\end{aligned}
$$

\section{Acknowledgment}

This work is sponsored by the NSFC (11101335, 11026060, Gansu Provincial Department of Education Fund (1101-02), NWNU-KJCXGC (03-69).

\section{References}

[1] A. M. A. El-Sayed, "Nonlinear functional-differential equations of arbitrary orders," Nonlinear Analysis. Theory, Methods \& Applications, vol. 33, no. 2, pp. 181-186, 1998.

[2] A. A. Kilbas, S. G. Samko, and O. I. Marichev, Fractional Integrals and Derivatives, Gordon and Breach Science Publishers, Yverdon, Switzerland, 1993.

[3] A. A. Kilbas and J. J. Trujillo, "Differential equations of fractional order: methods, results and problems. II," Applicable Analysis, vol. 81, no. 2, pp. 435-493, 2002. 
[4] A. A. Kilbas and J. J. Trujillo, "Differential equations of fractional order: methods, results and problems. I," Applicable Analysis, vol. 78, no. 1-2, pp. 153-192, 2001.

[5] K. S. Miller and B. Ross, An Introduction to the Fractional Calculus and Fractional Differential Equations, John Wiley \& Sons, New York, NY, USA, 1993.

[6] I. Podlubny, Fractional Differential Equations, vol. 198, Academic Press, San Diego, Calif, USA, 1999.

[7] K. S. Miller, "Fractional differential equations," Journal of Fractional Calculus, vol. 3, pp. 49-57, 1993.

[8] I. Podlubny, "The Laplace transform method for linear differential equations of the fractional order," UEF 02-94, Institute of Experimental Physics, Slovak Academy of Sciences , Kosice, Slovakia, 1994.

[9] A. Babakhani and V. Daftardar-Gejji, "Existence of positive solutions of nonlinear fractional differential equations," Journal of Mathematical Analysis and Applications, vol. 278, no. 2, pp. 434-442, 2003.

[10] D. Delbosco, "Fractional calculus and function spaces," Journal of Fractional Calculus, vol. 6, pp. 45-53, 1994.

[11] D. Delbosco and L. Rodino, "Existence and uniqueness for a nonlinear fractional differential equation," Journal of Mathematical Analysis and Applications, vol. 204, no. 2, pp. 609-625, 1996.

[12] V. Daftardar-Gejji and A. Babakhani, "Analysis of a system of fractional differential equations," Journal of Mathematical Analysis and Applications, vol. 293, no. 2, pp. 511-522, 2004.

[13] S. Q. Zhang, "The existence of a positive solution for a nonlinear fractional differential equation," Journal of Mathematical Analysis and Applications, vol. 252, no. 2, pp. 804-812, 2000.

[14] S. Q. Zhang, "Existence of positive solution for some class of nonlinear fractional differential equations," Journal of Mathematical Analysis and Applications, vol. 278, no. 1, pp. 136-148, 2003.

[15] Z. B. Bai and H. Lü, "Positive solutions for boundary value problem of nonlinear fractional differential equation," Journal of Mathematical Analysis and Applications, vol. 311, no. 2, pp. 495-505, 2005.

[16] Z. B. Bai, "On positive solutions of a nonlocal fractional boundary value problem," Nonlinear Analysis. Theory, Methods E Applications, vol. 72, no. 2, pp. 916-924, 2010.

[17] Y. Q. Wang, L. S. Liu, and Y. H. Wu, "Positive solutions for a nonlocal fractional differential equation," Nonlinear Analysis. Theory, Methods \& Applications, vol. 74, no. 11, pp. 3599-3605, 2011.

[18] M. A. Krasnoselskii, Positive Solutions of Operator Equations, P. Noordhoff Ltd., Groningen, The Netherlands, 1964.

[19] R. W. Leggett and L. R. Williams, "Multiple positive fixed points of nonlinear operators on ordered Banach spaces," Indiana University Mathematics Journal, vol. 28, no. 4, pp. 673-688, 1979. 


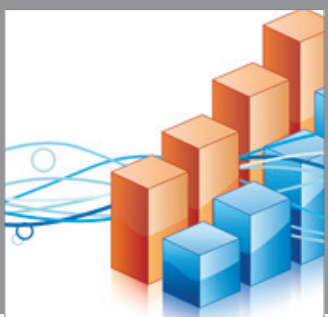

Advances in

Operations Research

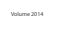

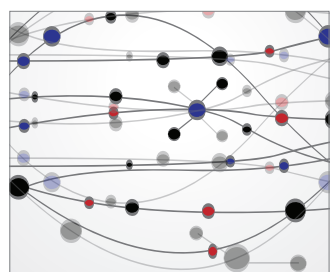

\section{The Scientific} World Journal
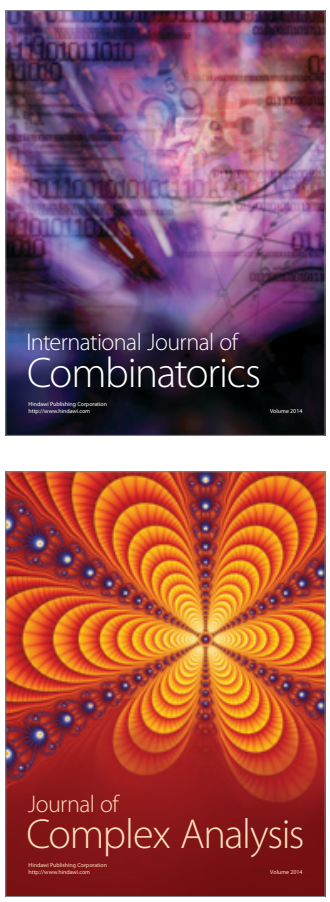

International Journal of

Mathematics and

Mathematical

Sciences
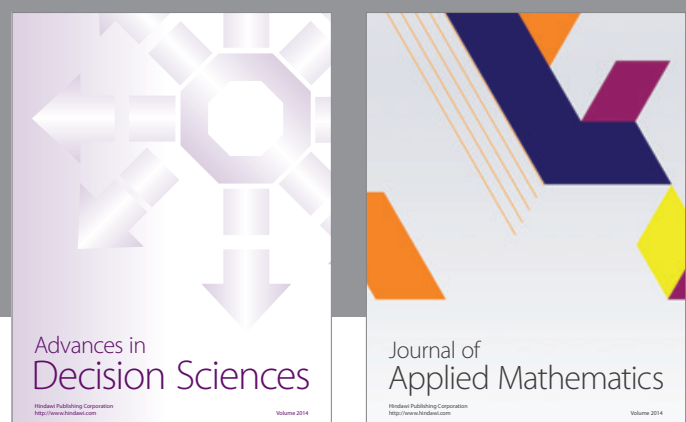

Journal of

Applied Mathematics
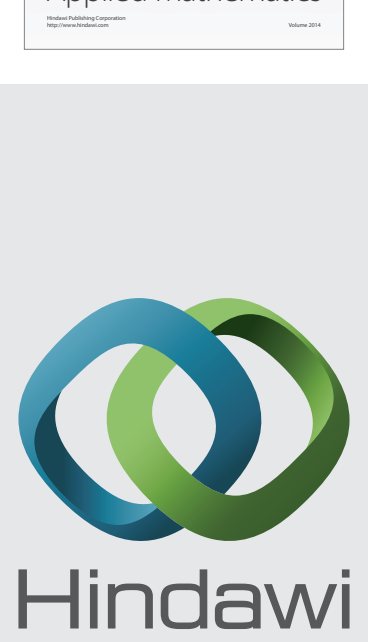

Submit your manuscripts at http://www.hindawi.com
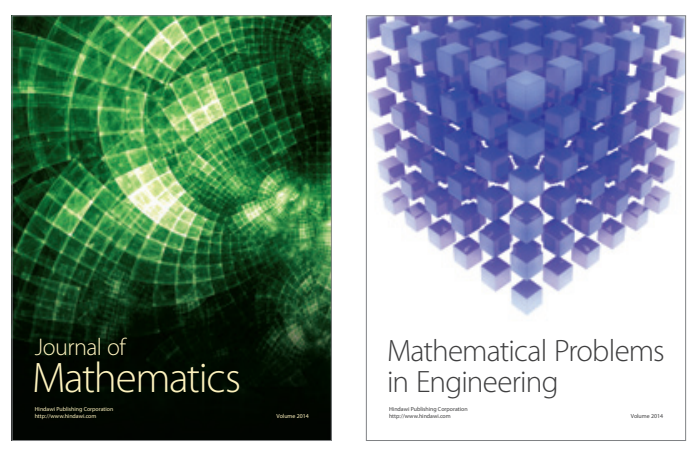

Mathematical Problems in Engineering
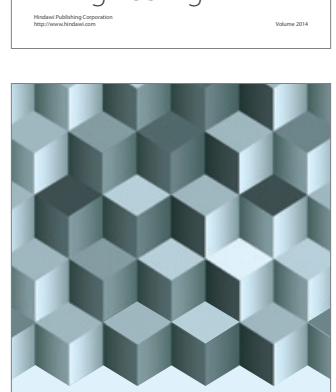

Journal of

Function Spaces
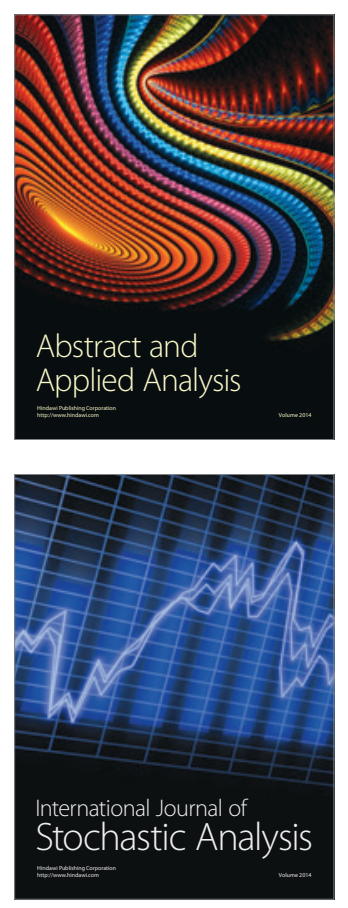

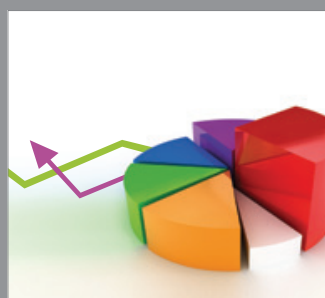

ournal of

Probability and Statistics

Promensencen
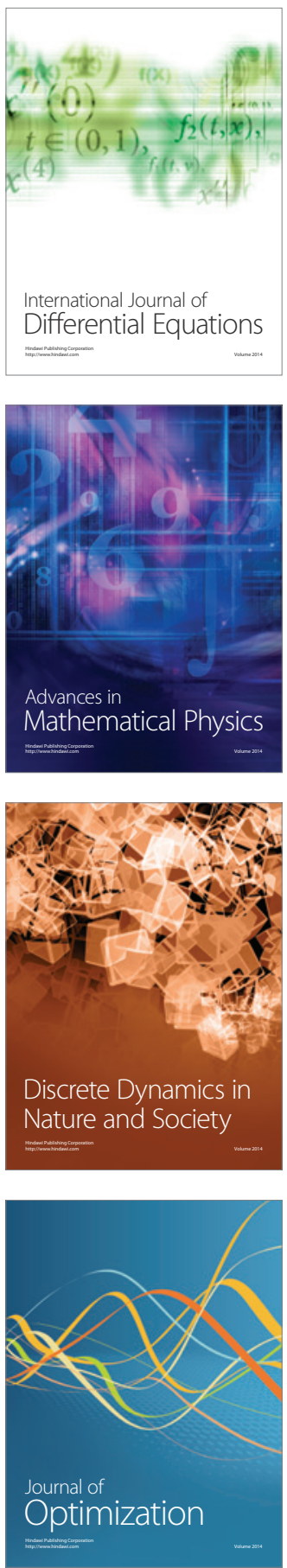